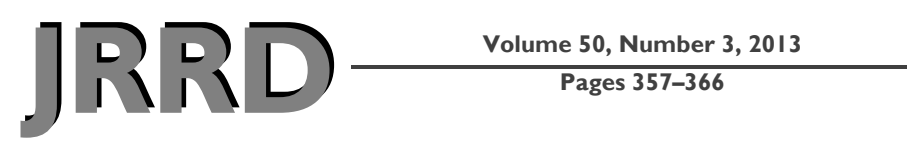

\title{
Bimanual gliding control for indoor power wheelchair driving
}

\author{
Yang-Hua Lin, PhD; ${ }^{1-2^{*}}$ Chung-Hsien Kuo, PhD; ${ }^{3}$ How-Hing Ng, MS; ${ }^{1}$ Wen-Yu Liu, PhD; ${ }^{1}$ Hen-Yu Lien, PhD ${ }^{1-2}$ \\ ${ }^{1}$ Department of Physical Therapy, College of Medicine, and ${ }^{2}$ Healthy Aging Research Center, Chang Gung University, \\ Tao-Yuan, Taiwan; ${ }^{3}$ Department of Electrical Engineering, National Taiwan University of Science and Technology, \\ Taipei, Taiwan
}

\begin{abstract}
Power wheelchairs are essential for many individuals with mobility impairment. The objective of this study was to investigate the effectiveness of bimanual gliding (BG) and conventional joystick (CJ) control in an indoor environment, with application to (1) wheelchair driving performance (i.e., practice time, completion time, and driving deviation) and (2) muscle activity of the upper limbs. This study included 22 participants (11 experienced manual wheelchair users and 11 novice manual wheelchair users). Experienced wheelchair users who used the BG strategy needed less time to practice and complete the task. Muscle activity of the upper limbs was focused on the triceps brachii, with relatively less use of the wrist muscles while applying the BG strategy. In novice wheelchair users, wrist muscles were less involved when using the BG control compared with the CJ control. The findings imply that it is feasible to modify manual wheelchairs using BG and motors, which can serve as an alternative option for wheelchair users.
\end{abstract}

Key words: control interface, driving assessment, electromyography, indoor wheelchair skill tests, muscle activity, power mobility, power wheelchair, spinal cord injury, upper limb, wheelchair.

\section{INTRODUCTION}

Mobility is an essential functional ability that is required for carrying out daily activities and social participation. Individuals with impaired bodily functions, such as patients with spinal cord injury (SCI), use wheelchairs to improve their mobility, quality of life, and social integration [1-3]. For these individuals who look for ways to decrease their physical burden, a power wheelchair is a better choice than a manual wheelchair. Commonly, a conventional joystick (CJ) is used as an interface to control the power wheelchair with one hand. Fehr et al. reported that up to 10 percent of individuals driving electrical power wheelchairs were unable to adequately operate a CJ [4], which requires approximately 1 to $2 \mathrm{~N}$ of force to operate [5]. The difficulty in driving electrical power wheelchairs was attributed to dexterity and strength impairments [4]. Pellegrini et al. reported that 47 out of 84 wheelchair users with restricted driving abilities because of Duchenne muscular dystrophy (DMD) had worse upper-limb performance than those with DMD whose driving abilities were not restricted, especially in key pinch strength [6]. However, the authors further reported that 18 restricted drivers with DMD and progressive

Abbreviations: $\mathrm{BB}=$ biceps brachii, $\mathrm{BG}=$ bimanual gliding, $\mathrm{CJ}=$ conventional joystick, $\mathrm{DC}=$ direct current, DMD = Duchenne muscular dystrophy, ECR = extensor carpi radialis, EMG = electromyography, FCR = flexor carpi radialis, $\mathrm{MVC}=$ maximal voluntary contraction, MWCU = manual wheelchair user, NMWCU = non-manual wheelchair user, RMS = root-meansquare, RMSE = root-mean-square error, SCI = spinal cord injury, $\mathrm{T}=$ thoracic, $\mathrm{TB}=$ triceps brachii.

*Address all correspondence to Yang-Hua Lin, PhD; Department of Physical Therapy, College of Medicine, Chang Gung University, 259 Wen-Hwa 1st Rd, Kwei-Shan, Tao-Yuan, Taiwan 333; +886-3-2118800; fax: +886-3-2118700.

Email: linyh@mail.cgu.edu.tw

http://dx.doi.org/10.1682/JRRD.2011.12.0230 
muscle weakness gradually lost their ability to drive with a CJ but regained unrestricted driving when using alternative control systems. Alternative interfaces for power wheelchairs have been designed that allow wheelchair control not only through the use of hands but also through the use of the brain, head, or tongue [7-10]. According to a biomechanical study by Finley et al. that explored wheelchair propulsion in 15 manual wheelchair users (MWCUs) with upper-limb impairment, the ability to propel the wheelchair with smaller peak joint angles and joint excursion of the wrist, elbow, and shoulder during the contact phase may protect users from developing secondary upper-limb pathologies [11].
Based on the possible functional joint limitations of the upper limbs in individuals with restricted mobility that may interfere with manual wheelchair propulsion, Kuo et al. developed a control interface called bimanual gliding (BG) [12], shown in Figure 1. In this interface, the gliding mechanism is installed on a modified manual wheelchair with two direct current (DC) brushless motors mounted on the wheel shafts and controlled by both hands on the slider handle. The gliding method is operated with the hand, which is not propelled by the upperlimb joints, in particular the shoulder joint. The BG control interface is operated by gliding forward and backward with both hands to control the driving direction or

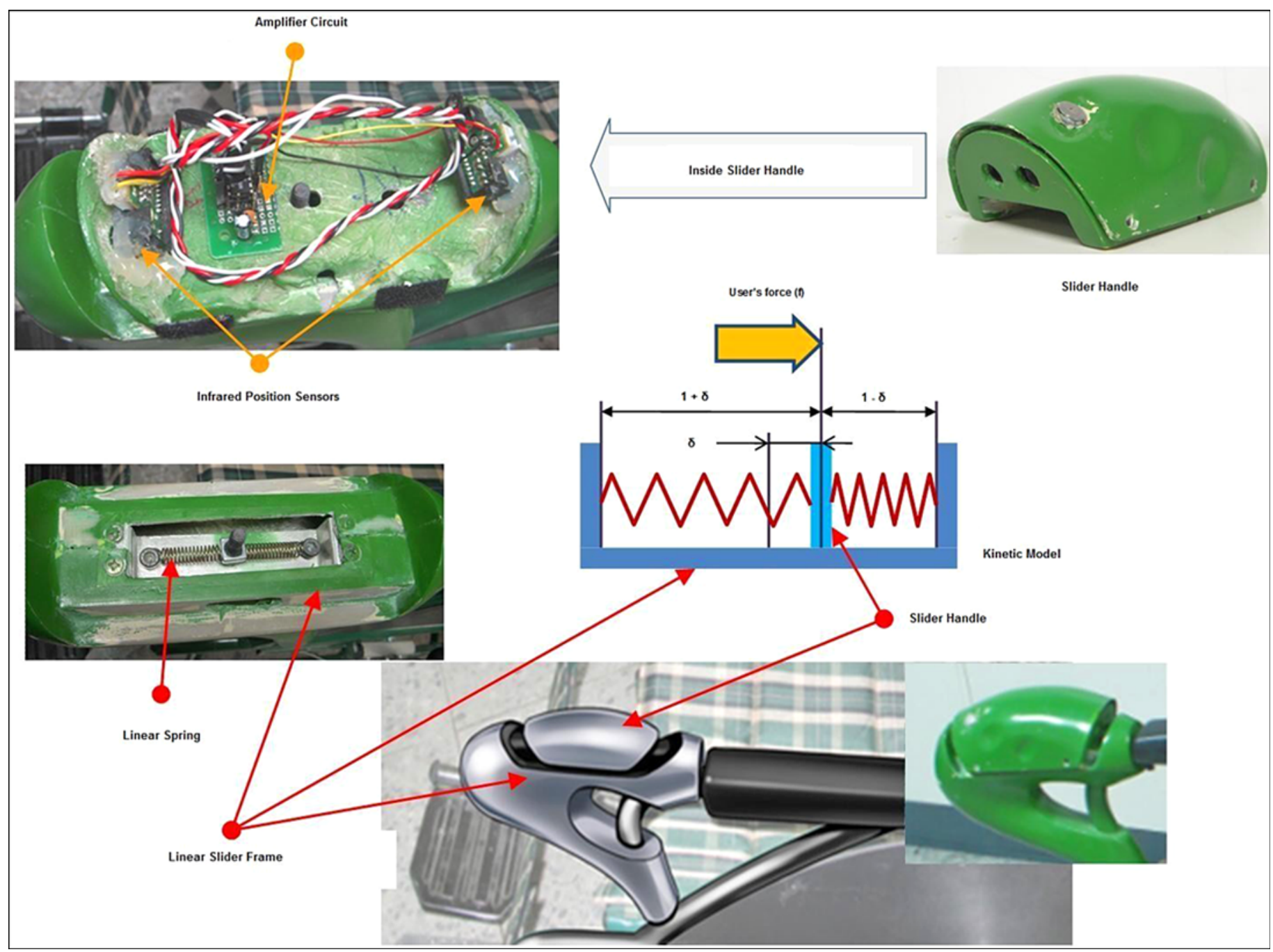

\section{Figure 1.}

Configuration of modified manual wheelchair with bimanual gliding control interface installed and two direct current brushless motors mounted on wheel shafts. 
by one hand to make turns. In a preliminary study comparing the use of BG and CJ with power wheelchairs, two out of five children demonstrated better symmetrical postural alignments while using the BG strategy [13]. In addition, a previous study that conducted a physiological evaluation of manually operated lever-propelled wheelchairs in one- and two-arm modes revealed that in unilateral arm use, the trunk muscles exert an additional effort during force production to stabilize the trunk in the transverse plane and to prevent axial rotation. This contributes to an increased heart rate and a higher oxygen uptake cost than that observed during the bimanual lever mode at a similar level of power output [14]. Nevertheless, until now there have been no reports on the maneuvering of power wheelchairs with unilateral versus bilateral control. Moreover, it has been suggested that bimanual movements might create better control and planning but require more computational time than the use of one hand alone [15]. Thus, the bimanual maneuver mode could be beneficial for power wheelchair users for postural alignment and better control.

The design concept of the wheelchair powered with a BG control interface used in the study was derived from manual wheelchair propulsion using both hands. We expected that MWCUs could drive intuitively by translating the propulsion experience to the bimanual maneuver. Few studies discuss the effect of the MWCU's experience on propulsion performance. Patterson and Draper found that significant differences were obtained for physiological characteristics (propulsion efficiency, peak oxygen consumption, and energy input) and for techniquerelated characteristics (propulsion time, push angle, work per stroke) between nondisabled people and people who use wheelchairs [16]. However, the effect of wheelchair propulsion experience on power wheelchair driving and even on the control interface maneuverability remains unclear.

The purpose of this study was to investigate driving performance and muscle activity of the upper limbs while driving a power wheelchair using BG versus CJ control interfaces in individuals with and without experience using a manual wheelchair. We hypothesized that both in individuals with and without manual wheelchair experience (1) the driving performance of wheelchairs maneuvered with the BG interface would be similar to those maneuvered with CJ-type interfaces, (2) the muscle activity for individuals using BG would be different from that of individuals using $\mathrm{CJ}$, and (3) both driving perfor- mance and muscle activity of the upper limbs would be different between MWCUs and nondisabled persons with no previous experience operating a wheelchair (nonmanual wheelchair users [NMWCUs]) based on our design concept, which could favored the experienced MWCUs.

\section{METHODS}

\section{Participants}

Two groups of participants were recruited in this study: MWCUs with SCI and NMWCUs. We recruited MWCUs and nondisabled users as the population of novel users in this study to assess if user experiences influence the driving performance of power wheelchairs with the BG control interface. A sample of convenience was used to recruit participants. All participants met the inclusion criteria, which were as follows: age over $18 \mathrm{yr}$, upper-limb muscle strength graded as "good" by the manual muscle test [17], and the ability to maintain an upright seated posture for at least $3 \mathrm{~h}$ in order to perform the driving skills. Participants were excluded if they had any disqualifying medical conditions, were unable to hold the control interface, or were unable to answer the questionnaire or follow instructions.

\section{Control Interfaces for Power Wheelchairs}

Two kinds of control interfaces were used in this study for driving performance tests: (1) a prototype of the BG control interface, as shown in Figure 1, developed by one of the authors (C. H. Kuo) and installed on a commercially available manual wheelchair, and (2) a conventional right-hand short-handle $\mathrm{CJ}$ equipped on a power wheelchair (type EB-2111, Comfort Co; Chia-Yi, Taiwan). The BG control interface was designed to be installed on the bilateral armrests of the manual wheelchair and was to be operated by both hands using a gliding mechanism for driving the two DC brushless motors, which were mounted on the wheel shafts and equipped with a microcontroller to control the two rear wheels of the wheelchair separately. The reason for using a manual wheelchair instead of a power wheelchair was that we were able to modify the manual wheelchair as a prototype of the powered wheelchair, equipped with bilateral external gliding interfaces and two DC brushless motors mounted on the wheel shafts as a power source. In addition, the wheelchair was equipped with infrared sensor arrays to avoid 
collision and to detect the distance of obstacles in front of the wheelchair.

The modified manual wheelchair with the BG (44 kg) is lighter than the commercial power wheelchair (68 kg). The dimensions of the wheelchair with the BG $(104 \times 64 \times$ $90 \mathrm{~cm})$ are different that of conventional power wheelchair $(102 \times 69 \times 75 \mathrm{~cm})$.

\section{Electromyography}

Active bipolar electrodes were used (model TSD 150 A, BIOPAC Systems Inc; Santa Barbara, California), with an interelectrode distance (center to center) of $3.5 \mathrm{~cm}$. The BIOPAC MP150 system was used to record the muscle activity of the upper limbs (biceps brachii [BB], triceps brachii [TB], flexor carpi radialis [FCR], and extensor carpi radialis [ECR]). After skin preparation, electromyography (EMG) electrodes were placed $2 \mathrm{~cm}$ proximal to the distal tendon and parallel to the muscle fiber of BB, midway between the posterior fold of the axillar and lateral epicondyle of the humerus of the TB, $5 \mathrm{~cm}$ from the medial epicondyle along the longitudinal axis of the forearm for FCR, one-third of the distance from the head of the ulna to the olecranon of the wrist and finger extensor muscles, and over the left wrist ulnar styloid process as ground electrodes. Data were acquired at a sampling frequency of $1,000 \mathrm{~Hz}$ and analyzed using the BIOPAC Acknowledge version 4.0. The signals were analog processed with a differential amplifier (bandwidth $=$ 50-500 Hz, input impedance $=1 \mathrm{G}$, common mode rejection ratio $=95 \mathrm{~dB}$ at $60 \mathrm{~Hz}$, and gain $=1,000)$. Each EMG file was passed through standard signal-processing techniques, including a filter to remove the average signal value and to remove any signal offset, a pass Butterworth filter with a corner frequency of $60 \mathrm{~Hz}$ to remove noise in the signal from the cardiac muscle, and a rootmean-square (RMS) low-pass filter (window length = $0.15 \mathrm{~s}$, window overlap $=0.075 \mathrm{~s}$ ). Upon completion of all tasks for a given subject, the raw EMG signal was converted to an RMS value. The mean RMS value was normalized by maximum voluntary contraction (MVC) recordings and used to scale each EMG. Prior to the experiment, the MVC for each muscle was obtained by a procedure in which each subject performed two $5 \mathrm{~s}$ MVCs at a $90^{\circ}$ elbow angle with a $45 \mathrm{~s}$ rest period between contractions.

\section{Driving Performance}

The driving task in this study was performed in an indoor square space $(6.60 \times 5.10 \mathrm{~m})$, with the driving routes consisting of $5 \mathrm{~m}$ straight lines and right/left turning points (Figure 2). The testing protocol included three tasks: (1) driving in a straight line, (2) making a left turn, and (3) making a right turn. The tasks were performed in a randomized order for a total of three trials for each task. Participants controlled their speed using either the CJ or BG excursion only and were encouraged to drive as quickly as possible to complete the requested task in order to maintain a consistent high degree of motivation. A webcam (QuickCam Ultra Vision, Logitech; Newark, California) and a laser pointer were installed under the seat of both the manual wheelchair with BG interface and the power wheelchair with $\mathrm{CJ}$ at a height of $0.4 \mathrm{~m}$ from the ground in order to record the pathways driven in the tests for analyzing driving deviation. To become acquainted with the indoor driving tasks, the participants practiced until their driving path deviation (RMS error [RMSE]) was within $10 \mathrm{~cm}$ from the center of the straight line. Task practice time, time to completion, and driving deviation were collected as performance measures. The completion time was determined as the time taken to move from the start line to the end line, as shown in Figure 2. Driving deviation was calculated as the average absolute distance from the middle line of the test track, according to a laser marker recorded with webcam, and shown as RSME. The task practice time was defined as the total duration taken by the participants to practice driving with each control interface. Following the driving

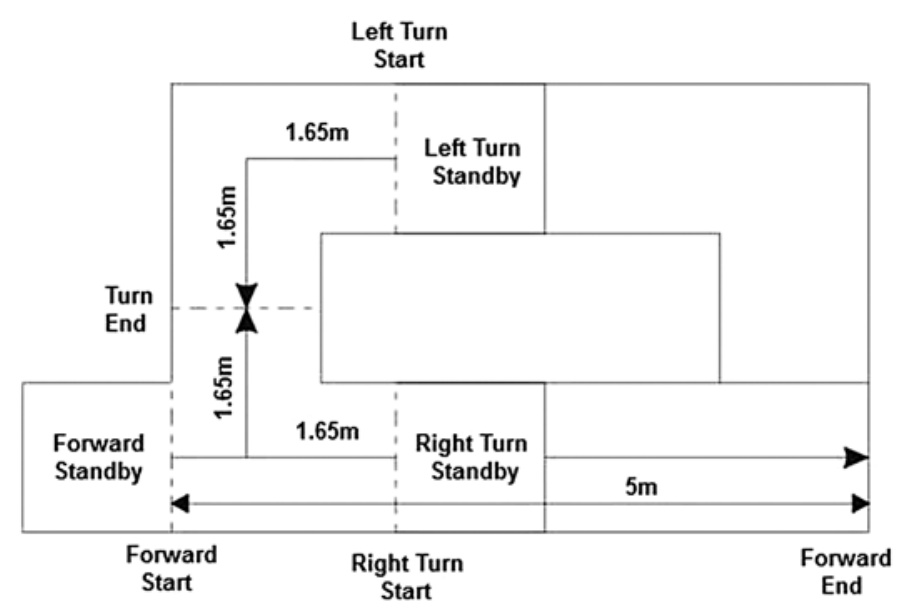

Figure 2.

Space layout for driving tasks. 
test, the participants were asked for their opinions regarding convenience and preference of driving interface type using the following questions: (1) Which one of the control interfaces do you feel was easiest to drive during the test in an indoor environment? (2) Which one of the control interfaces would you prefer to use to drive in an indoor environment?

Statistical analysis was performed using SPSS for Windows 10.0 (IBM; Armonk, New York). Means and standard deviations were the main descriptive measures. A two-way repeated measures analysis of variance (ANOVA) was used as an inference statistic to determine if there was any interaction effect for the group factor (MWCUs vs NMWCUs) and for interface type factor (BG vs CJ) and if there was any main effect for the statistical difference in performance measures (practice time, completion time, driving deviation, and muscle activation of the upper limbs) as dependent variables for each of the three driving tasks (straight line, left turn, right turn, respectively). Before using parametric testing, we checked the assumptions of normally distributed data and homogeneity of variance. All alpha values were set at 0.05 .

\section{RESULTS}

Twenty-two participants, 11 in each group, completed the indoor driving performance assessment. The MWCUs in the study had an SCI level ranging from thoracic (T)4 to T12 (Table 1), which indicated that they had intact innervations to the muscles of the upper limbs, in particular the ECR and FCR of the forearm musculature. Moreover, muscle strength of all participants was graded as "good" by the manual muscle test. The participants in the MWCU group had more than a year of experience using a manual wheelchair, and two of them also had experience driving a power wheelchair. Although these two participants had prior experience using power wheelchairs, they performed most of their daily activities using manual wheelchairs. The average age of participants ( 5 males, 6 females) in the NMWCU group was $25.3 \pm 3.2 \mathrm{yr}$, and the average body weight was $59.1 \pm 10.0 \mathrm{~kg}$. The participants in the MWCU group were significantly older than those in the NMWCU group $(p=0.01)$. All participants were right-handed.

\section{Driving Performance}

The participants spent an average of $10.3 \pm 6.3 \mathrm{~min}$ practicing using the BG control interface (MWCUs: $7.6 \pm$ $5.0 \mathrm{~min}$; NMWCUs: $13.1 \pm 6.5 \mathrm{~min}$ ) and $10.8 \pm 6.4 \mathrm{~min}$ using the CJ control interface (MWCUs: $9.2 \pm 4.1 \mathrm{~min}$, NMWCUs: $12.4 \pm 8.0 \mathrm{~min}$ ). There was no interaction between the groups and control interfaces in practice time ( $F=0.357, p=0.55)$. The NMWCU group took almost twice as long as the MWCUs in practice time to become familiar with the BG control interface $(p=0.02)$.

The interaction effect between the groups and the type of control interface affected task completion time and driving speed during the driving tests $(p<0.05)$, with the exception of the left turn, which was affected by the

Table 1.

Participant demographic characteristics.

\begin{tabular}{|c|c|c|c|c|c|c|c|}
\hline $\begin{array}{l}\text { Wheelchair User } \\
\quad(n=11)\end{array}$ & Sex & Age (yr) & Weight (kg) & $\begin{array}{c}\text { Neurological } \\
\text { Level }\end{array}$ & AIS & $\begin{array}{c}\text { Wheelchair } \\
\text { Experience (yr) }\end{array}$ & $\begin{array}{c}\text { Wheelchair } \\
\text { Type }\end{array}$ \\
\hline 1 & $\mathrm{~F}$ & 30 & 62 & T12 & A & 2 & MW \\
\hline 2 & $\mathrm{~F}$ & 32 & 62 & $\mathrm{~T} 11$ & A & 4 & MW \\
\hline 3 & F & 45 & 70 & $\mathrm{~T} 8$ & $\mathrm{C}$ & 15 & MW \\
\hline 4 & M & 23 & 58 & $\mathrm{~T} 11$ & A & 3 & MW \\
\hline 5 & M & 24 & 53 & T9 & A & 10 & MW \\
\hline 6 & M & 28 & 84 & $\mathrm{~T} 4$ & A & 10 & MW \\
\hline 7 & M & 28 & 56 & $\mathrm{~T} 11$ & A & 8 & MW \\
\hline 8 & M & 30 & 51 & $\mathrm{~T} 12$ & C & 2 & MW/P \\
\hline 9 & M & 32 & 47 & T9 & A & 11 & MW \\
\hline 10 & M & 41 & 55 & $\mathrm{~T} 12$ & C & 2 & MW/P \\
\hline 11 & M & 43 & 90 & $\mathrm{~T} 12$ & B & 3 & MW \\
\hline Average & - & $32.4 \pm 7.5$ & $62.5 \pm 13.6$ & - & - & $6.4 \pm 4.6$ & - \\
\hline
\end{tabular}

AIS = American Spinal Injury Association Impairment Scale, $\mathrm{F}=$ female, $\mathrm{M}=$ male, $\mathrm{MW}=$ manual wheelchair, $\mathrm{P}=$ power wheelchair, $\mathrm{T}=$ thoracic. 
JRRD, Volume 50, Number 3, 2013

Table 2.

Driving performance of experienced and inexperienced wheelchair users with two types of control interface.

\begin{tabular}{|c|c|c|c|c|c|}
\hline \multirow{2}{*}{ Measure } & \multirow{2}{*}{ Direction } & \multicolumn{2}{|c|}{ MWCU } & \multicolumn{2}{|c|}{ NMWCU } \\
\hline & & BG & CJ & BG & CJ \\
\hline Practice Time (min) & - & $7.6 \pm 5.0^{*}$ & $9.17 \pm 4.1$ & $13.1 \pm 6.5$ & $12.4 \pm 8.0$ \\
\hline \multirow[t]{2}{*}{ Completion Time (s) } & Forward & $31.9 \pm 0.6^{* \dagger}$ & $13.0 \pm 1.1^{*}$ & $33.2 \pm 1.0^{\dagger}$ & $16.9 \pm 0.8$ \\
\hline & Right turn & $24.9 \pm 1.3^{* \dagger}$ & $16.2 \pm 2.3$ & $29.9 \pm 1.4^{\dagger}$ & $15.4 \pm 1.5$ \\
\hline Speed (km/h) & Forward & $0.6 \pm 0.01^{* \dagger}$ & $1.4 \pm 0.1^{*}$ & $0.5 \pm 0.02^{\dagger}$ & $1.1 \pm 0.1$ \\
\hline \multirow[t]{3}{*}{ Driving Deviation (cm) } & Forward & $3.2 \pm 0.5$ & $3.0 \pm 0.5$ & $3.3 \pm 0.88$ & $3.00 \pm 0.3$ \\
\hline & Left turn & $3.1 \pm 0.5$ & $3.2 \pm 0.7$ & $3.0 \pm 0.8$ & $3.1 \pm 0.8$ \\
\hline & Right turn & $3.0 \pm 0.4$ & $3.4 \pm 0.6$ & $3.4 \pm 0.69$ & $3.4 \pm 0.8$ \\
\hline
\end{tabular}

Note: Values are mean \pm standard deviation.

${ }^{*}$ Significant difference between MWCU and NMWCU group, $p<0.05$ using independent $t$-test.

${ }^{\dagger}$ Significant difference between BG and CJ interface type, $p<0.05$ using paired $t$-test.

$\mathrm{BG}$ = bimanual gliding control interface, $\mathrm{CJ}=$ conventional joystick control interface, $\mathrm{MWCU}=$ manual wheelchair user, NMWCU = non-manual wheelchair user.

interface type only, as shown in Table 2. The MWCU group drove faster than the NMWCU group when using the BG in the forward direction and when making a right turn. The driving deviation did not demonstrate a significant difference between either of the groups and between the control interfaces during all three tests, as shown in Figure 3 (subject 2 of MWCUs).

\section{Muscle Activity of Upper Limbs}

There were no significant interactions between the groups and control interfaces with regard to muscle activity when participants either drove forward or made a left or right turn (Table 3 and Figure 4). The activation of the $\mathrm{BB}$ remained relatively constant regardless of the type of interface used. When using the BG control, the MWCUs demonstrated significantly less muscle activation of wrist flexors than when turning right using the $\mathrm{CJ}$ control ( $p<$ 0.05). When using the CJ control, the MWCUs used significantly greater TB activity than the NMWCUs while driving straight forward ( $p=0.003$ ); however, they used less wrist extensor activity to execute a right turn task. Similar to MWCUs, NMWCUs used significantly more muscle effort of the TB $(p<0.05)$ when using BG compared with using CJ. During turning tasks, NMWCUs apparently exerted less wrist muscle effort $(p<0.05)$ such as by wrist flexors during left turns and gave less muscle effort by both wrist flexors and wrist extensors during right turns. There was also more conspicuous muscle activity of the wrist extensors in the NMWCU group than in the MWCU group when using CJ to make a right turn.

\section{Preference}

Of the participants, 10 of the experienced MWCUs in this study preferred BG (MWCUs: 10, NMWCUs: 0) and 12 participants preferred CJ (MWCUs: 1, NMWCUs: 11). All of the participants self-reported that CJ was easier to operate; however, BG was more suitable in an indoor environment.

\section{DISCUSSION}

This study investigated the feasibility of using a BG control strategy for a modified manual wheelchair with motor power. Fehr et al. have reported that the physical impairment of an individual should be considered when assessing the adequacy of a control interface for a power wheelchair [4]. In the present study, the BG design was found to be feasible for indoor driving, which meets the requirement described by Finey et al. [11] for wheelchair users who have upper-limb impairment and who drive with smaller peak joint angles. Compared with the CJ control strategy, the BG control strategy requires less muscle activity of the forearm (wrist extensors and flexors) and the wrist angle to drive a power wheelchair. We found that driving deviation while driving using either control strategy was similar.

In the present study, we found that the muscle activity of the upper limbs when controlling a power wheelchair 
(a)

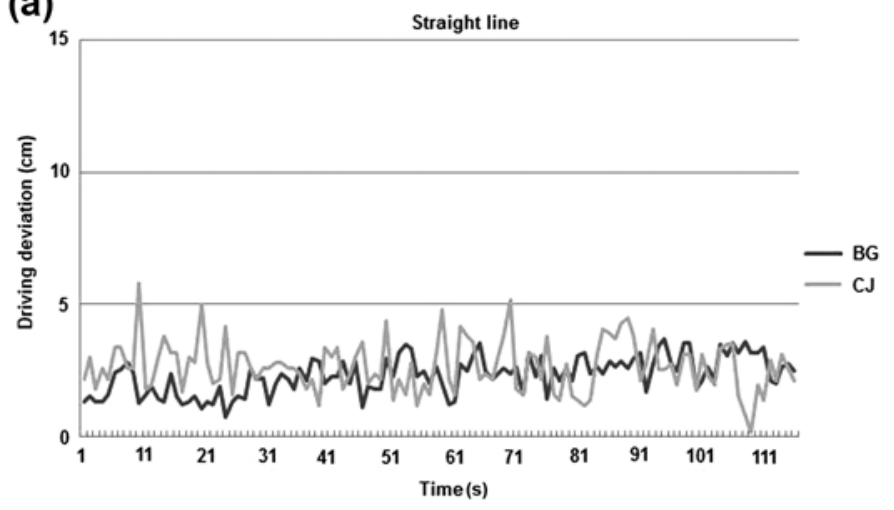

(b)

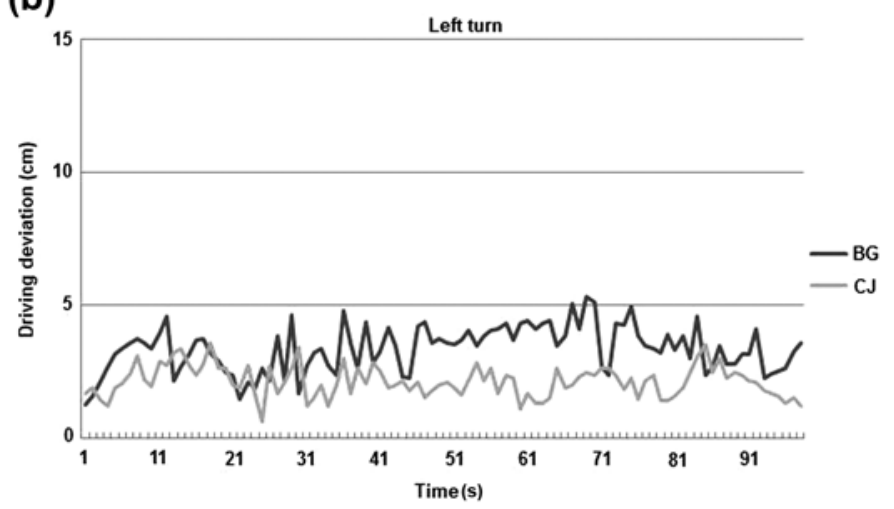

(c)

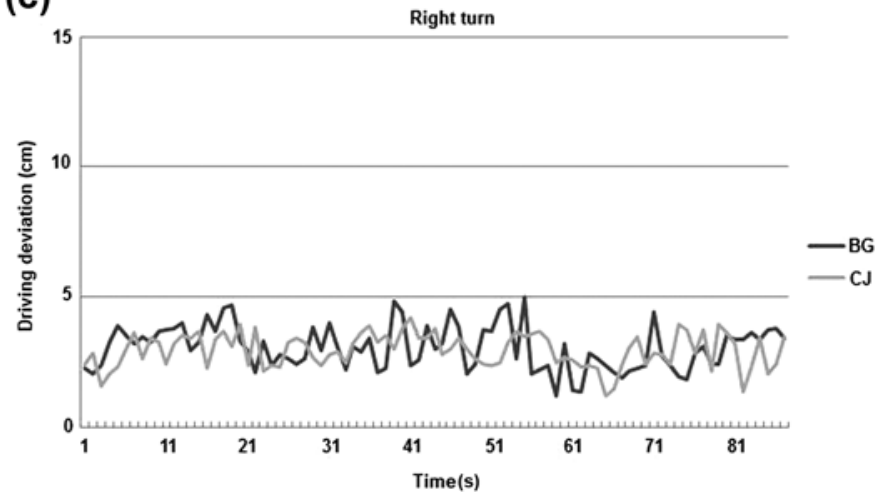

Figure 3.

Driving deviation of subject 2 in manual wheelchair user group: (a) straight line, (b) left turn, (c) right turn. BG = bimanual gliding, $\mathrm{CJ}=$ conventional joystick.

does depend on the types of strategies, bimanual or single maneuver, for the two types of control interfaces. Our study evaluated the muscle effort needed for indoor driving of a power wheelchair, which is an important issue for clinicians and could be useful as a reference for prescription. For operating wheelchairs equipped with the BG mechanism, the TB was found to be the predomi- nantly used muscle. For daily activity, BG may be an option for people with SCI who have preserved proximal muscles yet insufficient muscle strength in distal parts of the upper limbs. The muscles located in the forearm region responsible for wrist motion were apparently activated differently according to whether participants had experience using a manual wheelchair or not. Novice wheelchair users found that less skill was required to use the BG control interface than the CJ control interface and that BG did not require much muscle effort from the group of wrist muscles. It is known that overexertion of the forearm muscle may contribute to carpal tunnel syndrome of the wrist [18].

Most of the participants were novices at using power wheelchairs. In the MWCU group, participants had experience using manual wheelchairs but not power wheelchairs, with the exception of two participants who had experience using both. However, according to their selfreports, the inexperienced NMWCUs preferred the CJ design, differing from the experienced MWCUs, who preferred the BG control strategy. Based on practice time measurements, we found that the experience of manual wheelchair propulsion allowed MWCUs to learn new skills during the driving practice when they were required to control the BG control interface quickly. Driving a wheelchair with the BG interface requires maneuvering with both upper limbs bilaterally. This technique is beneficial to MWCUs who are acquainted with coordination patterns of wheelchair propulsion that use both upper limbs bilaterally. The potential users of the BG interface are not only those who would consider driving a powered wheelchair by adding it to their own manual wheelchair, but also those who are interested in trying out the BG interface.

Furthermore, driving tasks performed using the BG control interface took almost twice as long as the tasks performed using the CJ control interface, which could be a result of the participants' adaptation to the driving skills required for the BG design's bilateral arm coordination. This could be attributed to the differences in maximal speed and acceleration between the two wheelchairs. For the indoor powered wheelchair in the study, because of safety considerations we could not match the maximal speed, maximal rotational speed, and acceleration of the BG with the commercial power wheelchair for indoor and outdoor use. The wheelchair with the BG interface in the study had been designed for indoor use so that the maximal speed was close to the minimal speed of a commercial 
JRRD, Volume 50, Number 3, 2013

Table 3.

Muscle activity (\% of maximum voluntary contraction) in experienced and inexperienced wheelchair users while driving with two types of control interface.

\begin{tabular}{|c|c|c|c|c|c|}
\hline \multirow{2}{*}{ Direction } & \multirow{2}{*}{ Muscle } & \multicolumn{2}{|c|}{ MWCU } & \multicolumn{2}{|c|}{ NMWCU } \\
\hline & & BG & CJ & BG & CJ \\
\hline \multirow[t]{4}{*}{ Forward } & Biceps & $3.4 \pm 4.1$ & $3.2 \pm 5.5$ & $2.7 \pm 2.4$ & $1.5 \pm 0.7$ \\
\hline & Triceps & $13.2 \pm 5.4$ & $9.6 \pm 7.5^{*}$ & $14.2 \pm 11.2^{\dagger}$ & $3.7 \pm 2.9$ \\
\hline & FCR & $6.7 \pm 5.5$ & $4.9 \pm 5.4$ & $2.9 \pm 2.2$ & $4.6 \pm 2.5$ \\
\hline & ECR & $4.2 \pm 2.4$ & $7.0 \pm 3.6$ & $5.5 \pm 2.3$ & $9.1 \pm 5.9$ \\
\hline \multirow[t]{4}{*}{ Left Turn } & Biceps & $3.3 \pm 4.78$ & $3.4 \pm 4.1$ & $2.6 \pm 1.9$ & $2.1 \pm 1.9$ \\
\hline & Triceps & $11.3 \pm 4.3$ & $9.3 \pm 7.3$ & $12.9 \pm 9.7$ & $6.9 \pm 6.5$ \\
\hline & FCR & $4.6 \pm 6.4^{\dagger}$ & $5.8 \pm 4.5$ & $3.2 \pm 2.8^{\dagger}$ & $8.2 \pm 4.1$ \\
\hline & ECR & $6.0 \pm 5.16$ & $6.9 \pm 4.1$ & $7.3 \pm 5.1$ & $8.0 \pm 5.1$ \\
\hline \multirow[t]{4}{*}{ Right Turn } & Biceps & $2.7 \pm 1.4$ & $3.5 \pm 3.88$ & $2.4 \pm 1.4$ & $1.6 \pm 1.0$ \\
\hline & Triceps & $12.8 \pm 5.9$ & $9.2 \pm 6.8$ & $8.8 \pm 4.5$ & $8.4 \pm 9.2$ \\
\hline & FCR & $3.5 \pm 4.2^{\dagger}$ & $8.7 \pm 6.5$ & $3.1 \pm 3.0^{\dagger}$ & $14.4 \pm 7.8$ \\
\hline & ECR & $4.9 \pm 4.9$ & $6.4 \pm 5.5^{*}$ & $5.6 \pm 2.3^{\dagger}$ & $14.6 \pm 9.9$ \\
\hline
\end{tabular}

Note: Values are mean \pm standard deviation.

*Significant difference between MWCU and NMWCU group, $p<0.05$ using independent $t$-test.

${ }^{\dagger}$ Significant difference between BG and CJ interface type, $p<0.05$ using paired $t$-test.

$\mathrm{BG}=$ bimanual gliding control interface, $\mathrm{CJ}=$ conventional joystick control interface, $\mathrm{ECR}=$ extensor carpi radialis, FCR = flexor carpi radialis, $\mathrm{MWCU}=$ manual wheelchair user, NMWCU = non-manual wheelchair user.

power wheelchair $(\sim 4.5 \mathrm{~km} / \mathrm{h})$. However, in the pilot study, we adjusted the driving speed for the indoor driving environment, which apparently influenced the driving performance.

The limitations of our study are as follows: (1) this driving assessment was limited to a ground-level indoor environment, (2) the BG design was not applied to power wheelchairs and was not tested by power wheelchair users, (3) the subjects were all young users, and (4) backward driving with the BG design was enabled but not used as part of the test protocol. Experienced power wheelchair users would potentially use the proposed device, and their feedback will be valuable for further development. The BG interface used in this study can achieve backward driving; however, because driving safety was a priority, backward driving was not tested. The gliding direction of BG is the same as the driving direction of the wheelchair, and stopping or parking the wheelchair corresponds to the neutral position. Backward driving using the BG control interface needs further investigation, particularly with regard to the features of the muscles that are activated during backward driving.

In the present study, we did not measure the force required to control the bimanual gliding control interface. Although we found that less muscle activity is required to use the BG control interface, we need to further determine the relationship between muscle strength and force exertion as a variable to help assess the practicality of BG in clinical settings. In addition, our study was an assessment of indoor wheelchair driving using the BG and CJ control interfaces in a restricted space within a short period of time. It will also be necessary to determine the daily demands of indoor driving, which require activating the controller for extended periods during daily use, and to consider the cumulative influence of these demands on comfort and preference of control interface.

Compared with the CJ, the new BG device incurs additional costs from more wiring and the installation of motors for bilateral translational gliding signals on the BG control interface. The cost of a pair of BG sensors is estimated at around US\$35, without the motors and microcontroller included. Furthermore, our study presents the BG control interface as a prototype, and it should be validated for technical or mechanical issues.

\section{CONCLUSIONS}

The BG control strategy can be considered as an alternative control interface for power wheelchairs. The BG control strategy required less muscle activity of the forearm (wrist extensors and flexors) to drive a power wheelchair than the CJ control strategy. Experienced MWCUs displayed reduced task completion time and 

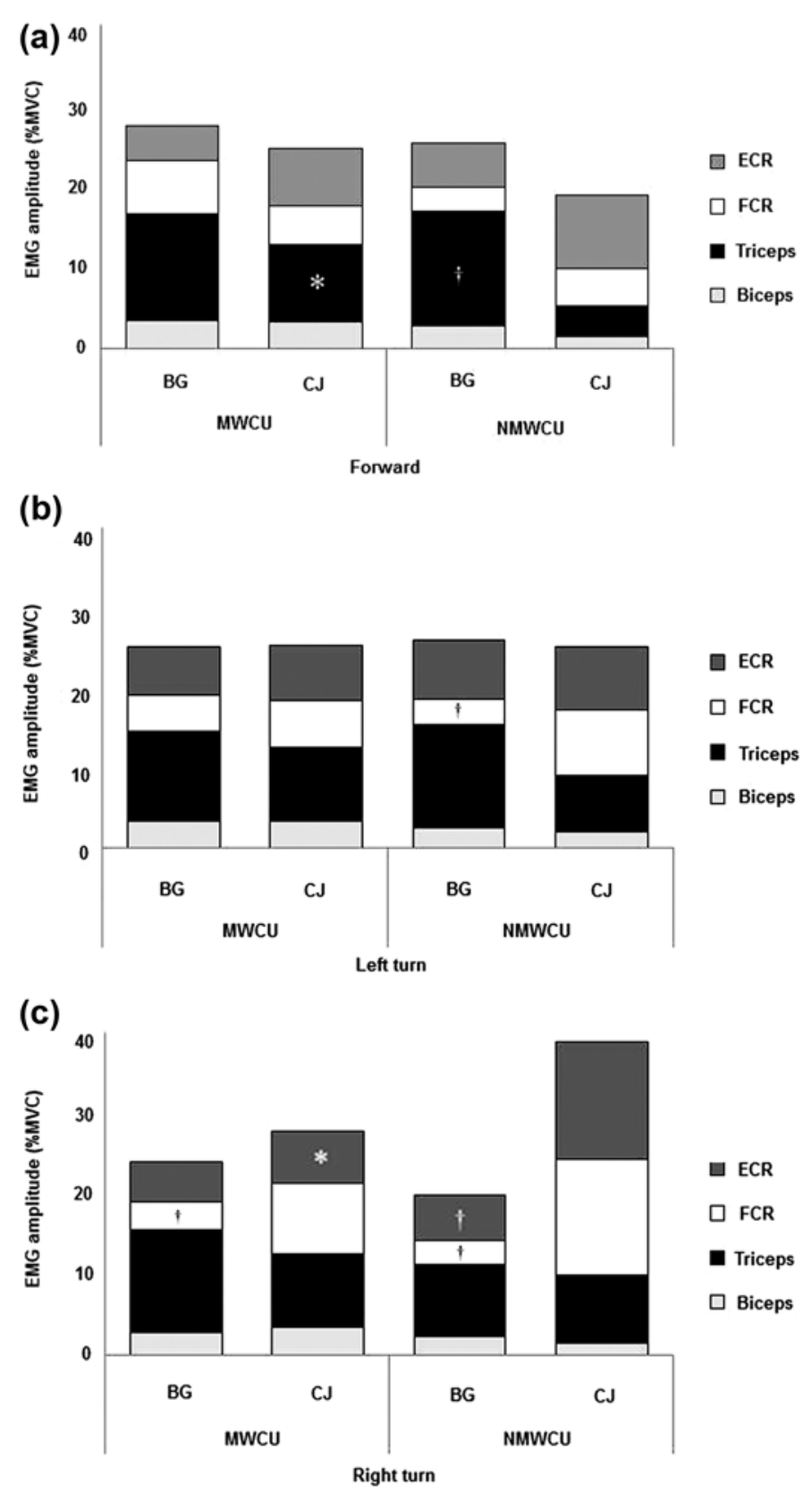

Figure 4.

Muscle activation of upper limb while driving powered wheelchair (a) forward, (b) making a left turn, and (c) making a right turn with two types of control interface (bimanual gliding [BG] and conventional joystick [CJ]). * Significant differences between MWCU and NMWCU group, $p<0.05$ using independent $t$-tests. ${ }^{\dagger}$ Significant difference between BG and CJ group, $p<0.05$ using paired $t$-tests. ECR = extensor carpi radialis, EMG = electromyography, FCR = flexor carpi radialis, MVC = maximal voluntary contraction, MWCU = manual wheelchair user; NMWCU = non-manual wheelchair user. muscle activity of the forearm while using the BG control interfaces compared with their novice counterparts. Further research efforts should be directed toward assessing the feasibility of power wheelchairs with bimanual control interfaces for individuals with different levels of SCI.

\section{ACKNOWLEDGMENTS}

\section{Author Contributions:}

Study concept and design: Y. H. Lin, C. H. Kuo.

Acquisition of data: H. H. Ng.

Analysis and interpretation of data: H. H. Ng, W. Y. Liu, H. Y. Lien. Drafting of manuscript: Y. H. Lin, H. H. Ng.

Critical revision of manuscript for important intellectual content:

Y. H. Lin, C. H. Kuo, H. Y. Lien, W. Y. Liu.

Obtained funding: C. H. Kuo, Y. H. Lin.

Financial Disclosures: The authors have declared that no competing interests exist.

Funding/Support: This article was based on work supported in part by the National Science Council, Republic of China (Taiwan) (grants NSC 97-3114-e-011-002 and NSC 98-2218-E-011-017).

Institutional Review: This study was approved by the Institutional Review Board of Chang Gung Medical Foundation, Taiwan. All participants provided informed consent.

Participant Follow-Up: The authors have no plans to notify the study subjects of the publication of this article because of a lack of contact information.

\section{REFERENCES}

1. Brandt A, Iwarsson S, Ståhle A. Older people's use of powered wheelchairs for activity and participation. J Rehabil Med. 2004;36(2):70-77. [PMID:15180221] http://dx.doi.org/10.1080/16501970310017432

2. Routhier F, Vincent C, Desrosiers J, Nadeau S. Mobility of wheelchair users: a proposed performance assessment framework. Disabil Rehabil. 2003;25(1):19-34. [PMID:12554389]

3. Chan SC, Chan AP. User satisfaction, community participation and quality of life among Chinese wheelchair users with spinal cord injury: a preliminary study. Occup Ther Int. 2007;14(3):123-43. [PMID:17624872] http://dx.doi.org/10.1002/oti.228

4. Fehr L, Langbein WE, Skaar SB. Adequacy of power wheelchair control interfaces for persons with severe disabilities: a clinical survey. J Rehabil Res Dev. 2000;37(3): 353-60. [PMID:10917267]

5. Dicianno BE, Spaeth DM, Cooper RA, Fitzgerald SG, Boninger ML. Advancements in power wheelchair joystick technology: Effects of isometric joysticks and signal conditioning on driving performance. Am J Phys Med Rehabil. 
2006;85(8):631-39. [PMID:16865017]

http://dx.doi.org/10.1097/01.phm.0000228519.54763.b4

6. Pellegrini N, Guillon B, Prigent H, Pellegrini M, Orlikovski D, Raphael JC, Lofaso F. Optimization of power wheelchair control for patients with severe Duchenne muscular dystrophy. Neuromuscul Disord. 2004;14(5): 297-300. [PMID:15099587] http://dx.doi.org/10.1016/j.nmd.2004.02.005

7. Corrigan R, McBurney H. Community ambulation: environmental impacts and assessment inadequacies. Disabil Rehabil. 2008;30(19):1411-19. [PMID:18720122] http://dx.doi.org/10.1080/09638280701654542

8. Wee J, Lysaght R. Factors affecting measures of activities and participation in persons with mobility impairment. Disabil Rehabil. 2009;31(20):1633-42. [PMID:19479498] http://dx.doi.org/10.1080/09638280902736346

9. Mortenson WB, Miller WC, Miller-Pogar J. Measuring wheelchair intervention outcomes: development of the wheelchair outcome measure. Disabil Rehabil Assist Technol. 2007;2(5):275-85. [PMID:19263533] http://dx.doi.org/10.1080/17483100701475863

10. Hu MH, Ko CC, Chai HM, Wu YT. A preliminary report of assistive device usage in the disabled. FJPT. 2004;29:396404.

11. Finley MA, Rasch EK, Keyser RE, Rodgers MM. The biomechanics of wheelchair propulsion in individuals with and without upper-limb impairment. J Rehabil Res Dev. 2004;41(3B):385-95. [PMID:15543456] http://dx.doi.org/10.1682/JRRD.2004.03.0385

12. Kuo CH, Chan YC, Chiou KW, Siao JW. Innovative wheelchair manipulation interface with collision avoidance and heart beat monitoring. Proceedings of the 6th International Conference on Ubiquitous Robots and Ambient Intelligence; 2009 Oct 29-30; Gwangju, Korea.

13. Liu WY, Chen FJ, Kuo CH, Lin YH, Cheng CH, Chen TS, Yu YJ. Preliminary comparisons of postural alignments of children with cerebral palsy using joystick and bimanual gliding access devices to drive the power wheelchair. FJPT. 2011;36(3):216-24.
14. van der Woude LH, Veeger HE, de Boer Y, Rozendal RH. Physiological evaluation of a newly designed lever mechanism for wheelchairs. J Med Eng Technol. 1993;17(6): 232-40. [PMID:8169940] http://dx.doi.org/10.3109/03091909309006331

15. Asai T, Sugimori E, Tanno Y. Two agents in the brain: motor control of unimanual and bimanual reaching movements. PLoS ONE. 2010;5(4):e10086. [PMID:20386749] http://dx.doi.org/10.1371/journal.pone.0010086

16. Patterson P, Draper S. Selected comparisons between experienced and non-experienced individuals during manual wheelchair propulsion. Biomed Sci Instrum. 1997;33:477-81. [PMID:9731406]

17. Hislop HJ, Montgomery J. Daniels and Worthingham's muscle testing: Techniques of manual examination. St. Louis (MO): Saunders/Elsevier; 2007.

18. Wieslander G, Norbäck D, Göthe CJ, Juhlin L. Carpal tunnel syndrome (CTS) and exposure to vibration, repetitive wrist movements, and heavy manual work: a case-referent study. Br J Ind Med. 1989;46(1):43-47. [PMID:2920142]

Submitted for publication December 4, 2011. Accepted in revised form August 21, 2012.

This article and any supplementary material should be cited as follows:

Lin YH, Kuo CH, Ng HH, Liu WY, Lien HY. Bimanual gliding control for indoor power wheelchair driving. J Rehabil Res Dev. 2013;50(3):357-66.

http://dx.doi.org/10.1682/JRRD.2011.12.0230

ResearcherID/ORCID: Yang-Hua Lin, PhD: C-7243-2013; Chung-Hsien Kuo, PhD: C-7253-2013; How-Hing Ng, MS: C-7370-2013; Wen-Yu Liu, PhD: C-7299-2013; Hen-Yu Lien, PhD: C-7444-2013

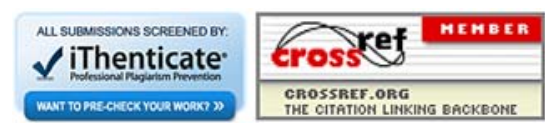

